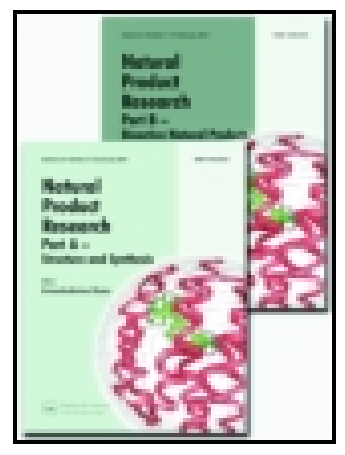

Natural Product Research

Formerly Natural Product Letters

ISSN: 1478-6419 (Print) 1478-6427 (Online) Journal homepage: http://www.tandfonline.com/loi/gnpl20

\title{
Caffeoylquinic acids from antiplasmodial active extract of Xanthium cavanillesii fruits and their molecular modelling studies
}

\author{
Alex Gutterres Taranto, Sônia Carine Cova Costa, Franco Henrique Andrade \\ Leite, Matheus Santos de Sá, Milena Botelho Pereira Soares, Miriam Moreira \\ Mussi \& Alexsandro Branco
}

To cite this article: Alex Gutterres Taranto, Sônia Carine Cova Costa, Franco Henrique Andrade Leite, Matheus Santos de Sá, Milena Botelho Pereira Soares, Miriam Moreira Mussi \& Alexsandro Branco (2016): Caffeoylquinic acids from antiplasmodial active extract of Xanthium cavanillesii fruits and their molecular modelling studies, Natural Product Research, DOI: $\underline{10.1080 / 14786419.2016 .1219856}$

To link to this article: http://dx.doi.org/10.1080/14786419.2016.1219856

+ View supplementary material $[\pi$

Submit your article to this journal $\pi$
Published online: 13 Aug 2016.

Џ Article views: 8 


\section{Caffeoylquinic acids from antiplasmodial active extract of Xanthium cavanillesii fruits and their molecular modelling studies}

\section{Alex Gutterres Taranto ${ }^{a}$ iD, Sônia Carine Cova Costab ${ }^{b}$ Franco Henrique Andrade Leite $^{b}$, Matheus Santos de Sác, Milena Botelho Pereira Soares ${ }^{c}$, Miriam Moreira Mussid and Alexsandro Branco ${ }^{b}$}

aLaboratório de Química Farmacêutica Medicinal, Universidade Federal de São João del-Rei, Divinópolis, Brazil; 'baboratórios de Fitoquímica e Modelagem Molecular, Universidade Estadual de Feira de Santana, Feira de Santana, Brazil; 'Centro de Pesquisas Gonçalo Moniz, Fundação Oswaldo Cruz, Salvador, Brasil; ' Laboratório de Química Faramcêutica, Universidade Católica de Pelotas, Pelotas, Brasil

\section{ABSTRACT}

The antiplasmodial active extract of Xanthium cavanillesii contains 3,4-dicaffeoyl quinic acid (3,4-DCQA), 3,5-dicaffeoyl quinic acid (3,5DCQA) and 1,3,5-tricaffeoyl quinic acid (1,3,5-TCQA). These results inspired us to investigate the interaction of these molecules with a promising validated target of Plasmodium, PfATP6 orthologue of mammalian $\mathrm{Ca}^{+2}$-ATPase. Models of this receptor were obtained through comparative modelling. Afterwards, molecular docking studies were used to identify possible interaction modes of these caffeoyl quinic derivatives on the binding site. The 1,3,5-TCQA had the best energy, but all of these had better energy than thapsigargin, a non-competitive inhibitor of the sarco/endoplasmatic reticulum $\mathrm{Ca}^{+2}$ ATPase (SERCA).
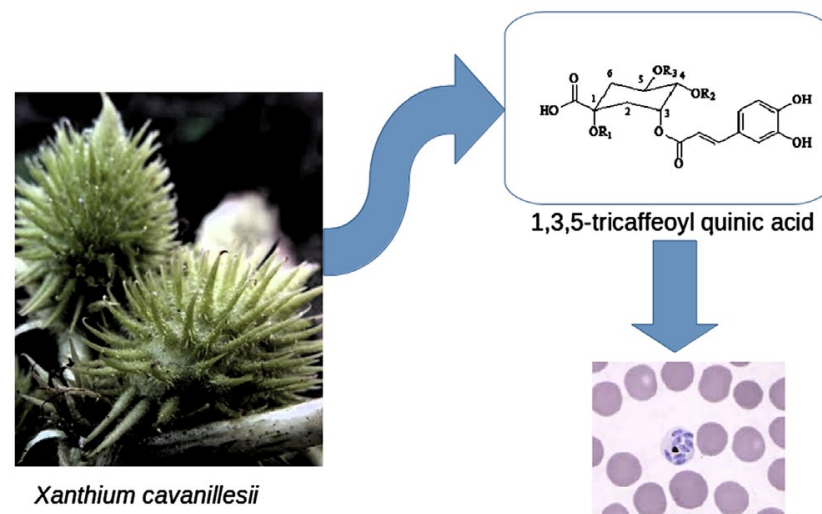

1,3,5-tricaffeoyl quinic acid

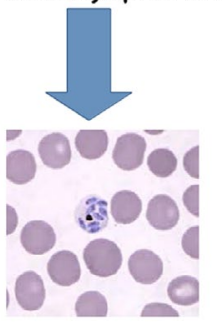

Antimalarial Assay

\section{ARTICLE HISTORY}

Received 22 October 2015

Accepted 6 July 2016

\section{KEYWORDS}

Xanthium cavanillesii; caffeoyl quinic acid; antiplasmodial activity; PfATPase 


\section{Introduction}

Malaria is an infectious, non-contagious disease with chronic evolution and acute manifestations affecting 97 countries and territories in tropical and subtropical areas worldwide. In 2013,198 million cases were reported leading to 584,000 deaths. It is more prevalent in lower and middle-income countries, with Africa being the most affected continent with $90 \%$ of the deaths occurring in children under five years (WHO 2014).

Xanthium cavanillesii (Asteraceae) is an herbaceous annual plant that is extremely competitive with other crops; it has been considered to be the worst weed on plantations. This plant is known for intoxicating several ruminant animals mainly in the south and south-east of Brazil. Toxicity generally occurs in spring and early summer due to the ingestion of seedlings in the cotyledonary stage. Seeds are also toxic and cause intoxication when the fruits are mixed with foodstuffs or hay (Mendez et al. 1998). Previous studies have shown that the compound responsible for this mortality is the diterpene kaurene called carboxyatractyloside (Obatomi \& Bach 1998; Chen et al. 2015).

In this study, we described the characterisation, the docking studies and the antimalarial assay (Mendiola et al. 2014; Mollinedo et al. 2015) of three caffeoyl quinic acid derivatives of an antimalarial active extract from $X$. cavanillesii (Figure 1). These structures have a caffeoylquinic acid scaffold (Manohar et al. 2013), inspiring us to perform docking studies

(A)<smiles>[Z10][Y16](=O)(CCCC)OC(=O)/C=C/c1ccc(O)c(O)c1</smiles>

$\begin{array}{llll} & \mathrm{R}_{1} & \mathrm{R}_{2} & \mathrm{R}_{3} \\ \mathbf{1} & \mathrm{H} & \mathrm{caf} & \mathrm{H} \\ \mathbf{2} & \mathrm{H} & \mathrm{H} & \mathrm{caf} \\ \mathbf{3} & \mathrm{caf} & \mathrm{H} & \mathrm{caf}\end{array}$

(B)

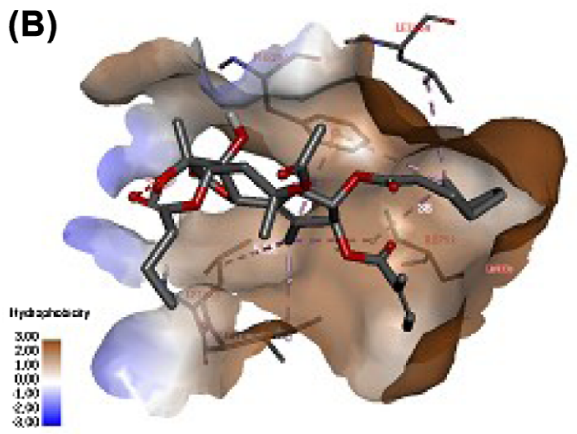

(C)

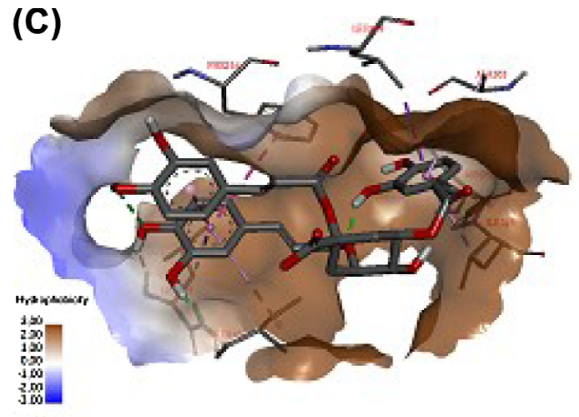

Figure 1. Caffeoyl quinic acid scaffold: (1) 3,4-dicaffeoyl quinic acid (3,4-DCQA); (2) 3,5-dicaffeoyl quinic acid (3,5-DCQA) and (3) 1,3,5-tricaffeoyl quinic acid (1,3,5-TCQA). 
on the PfATP6 model, which was previously constructed by comparative homology modelling (Guimarães et al. 2015). The experimental details are described in the Supplementary Material.

\section{Results and discussion}

The $X$. cavanillesii fruit extract was assayed in vitro against the W2 strain of Plasmodium falciparum, which showed antiplasmodial activity, with $\mathrm{IC}_{50}$ values of $65.8 \mu \mathrm{g} / \mathrm{mL}$, against artemisinin with $0.0022 \mu \mathrm{g} / \mathrm{mL}$. The phytochemical study indicated the possibility to obtain 3,4-DCQA (compound 1), 3,5-DCQA (2) and 1,3,5-TCQA (3) (Figure 1(A)). Structural elucidation of the isolated natural products was achieved through spectrometric analyses, such as hydrogen nuclear magnetic resonance and high performance liquid chromatography coupled to a mass spectrometry detector (HPLC-Msn, Figure S1) ). These compounds have not been previously reported in X. cavanillesii although they have already isolated from other species of the Xanthium (Mahmoud et al. 2005).

The above results inspired us to investigate the pharmacological mechanism by evaluating the affinity of these compounds against the PfATP6 model using docking routine (Trott \& Olson 2010). As a result, all caffeoylquinic acid derivatives had better binding energies than thapsigargin (-7.4 kcal/mol). Furthermore, 1,3,5-TCQA had the highest energy $(-8.6 \mathrm{kcal} / \mathrm{mol})$. Moreover, 3,4-DCQA and 3,5-DCQA had binding energies of -8.1 and $-7.7 \mathrm{kcal} / \mathrm{mol}$, respectively. These findings suggested that caffeoylquinic acid is a pharmacophoric moiety of this class of compounds.

In addition, thapsigargin could complex with the PfATP6 model through hydrophobic interactions with Phe254, Leu258, Ile752, Leu815 and Ile816 (Figure 1(B)). Similarly, 1,3,5TCQA could have hydrophobic interactions with Phe254, Leu258, Ala303, Asn755and Ile756. Furthermore, 1,3,5-TCQA could bind, as a hydrogen bond donor, to Leu815 and Ile816, increasing the affinity profile (Figure $1(C)$ ).

Finally, despite the fact that biological assays were not performed for isolated compounds, the experimental and in silico data have showed that quinic acid derivatives are promising antimalarial lead compounds and previously published studies have identified antimalarial properties on those compounds (Carbonara et al. 2012). Further assays to confirm and explain the biological properties of $X$. cavanillesii extracts are in progress.

\section{Experimental}

\subsection{Plant material}

X. cavanillesii was collected from São Lourenço do Sul, Rio Grande do Sul State, Brazil. A voucher specimen was deposited at the Herbarium of the Department of Biology of the State University of Feira de Santana (HUEFS 139439).

\subsection{Preparation of the ethanolic extract}

The fruits of $X$. cavanillesii $\left(3.9 \mathrm{~kg}\right.$ ) were manually separated, dried at $45^{\circ} \mathrm{C}$ and pulverised. Extraction commenced with ethyl acetate, which was aimed at removing the waxes, which was followed by the use of ethanol for $72 \mathrm{~h}$ at room temperature. The respective solvent was filtered and evaporated using a rotaevaporator apparatus, resulting in the ethanol $(50 \mathrm{~g})$ extract. 


\section{Conclusions}

The ethanolic extract of $X$. cavanillesii had significant activity against the $P$. falciparum W2 strain. The chemical investigation of this extract allowed for characterisation of 3,4-DCQA, 3,5-DCQA and 1,3,5-TCQA. Comparing the calculated structures, 1,3,5-TCQA had the same interaction pattern as thapsigargin. As a result, 1,3,5-TCQA can be used as a lead compound for further biological studies against PfATP6. These computational studies not only shed light on the mechanism of PfATP6 inhibition, but also provide insight for the rational improvement of inhibitory potency. Consequently, these strategies can be used to identify novel antimalarial drug candidates from natural sources.

\section{Supplementary material}

Experimental details relating to this article are available on line.

\section{Acknowledgements}

The authors wish to thank Prof Dr Rodrigo Bagueira (UFF) for technical assistance with the NMR analysis.

\section{Disclosure statement}

No potential conflict of interest was reported by the authors.

\section{Funding}

This work was supported by FAPESB [grant number SEMIÁRIDO - PET0018/2007]; CAPES [grant number PROCAD 2007]; FAPEMIG [grant number APQ-00557-14]; CNPq [grant number 475512/2010-3], [grant number 449984/2014-1]; PPGBiotec/UFSJ.

\section{ORCID}

Alex Gutterres Taranto (D) http://orcid.org/0000-0002-6086-1043

\section{References}

Carbonara T, Pascale R, Argentieri MP, Papadia P, Fanizzi FP, Villanova L, Avato P. 2012. Phytochemical analysis of a herbal tea from Artemisia annua L. J Pharm Biomed Anal. 62:79-86. Available from: http://www.ncbi.nlm.nih.gov/pubmed/22305080

Chen WH, Liu WJ, Wang Y, Song XP, Chen GY. 2015. A new naphtoquinone and other antibacterial constituents from the roots of Xanthium sibiricum. Nat Prod Res. 29:739-744.

Guimarães DSM, Fonseca AL, Batista R, Comar Junior M, Oliveira AB, Taranto AG, Varotti FDP. 2015. Structure-based drug design studies of the interactions of ent-kaurane diterpenes derived from wedelia paludosa with the Plasmodium falciparum sarco/endoplasmic reticulum Ca ${ }^{2+}$-ATPase PfATP6. Mem Inst Oswaldo Cruz. 110:255-258.

Mahmoud AA, Ahmed AA, Al-Shihry SS, Spring O. 2005. A new heterocyclic glucoside from the fruits of Xanthium pungens. Nat Prod Res. 19:585-589.

Manohar S, Khan SI, Kandi SK, Raj K, Sun G, Yang X, Calderon Molina AD, Ni N, Wang B, Rawat DS. 2013. Synthesis, antimalarial activity and cytotoxic potential of new monocarbonyl analogues of curcumin. Bioorg Med Chem Lett. 23:112-116. 
Mendez MC, dos Santos RC, Riet-Correa F. 1998. Intoxication by Xanthium cavanillesii in cattle and sheep in southern Brazil. Vet Hum Toxicol. 40:144-147.

Mendiola J, Regalado EL, Díaz-García A, Thomas OP, Fernández-Calienes A, Rodríguez H, Laguna A, Valdés O. 2014. In vitro antiplasmodial activity, cytotoxicity and chemical profiles of sponge species of Cuban coasts. Nat Prod Res. 28:312-317.

Mollinedo P, Vila JL, Arando H, Sauvain M, Deharo E, Bravo JA. 2015. Anti-infective assessment of Senecio smithioides (Asteraceae) and isolation of 9-oxoeuryopsin, a furanoeremophilane-type sesquiterpene with antiplasmodial activity. Nat Prod Res. 25:1-4.

Obatomi DK, Bach PH. 1998. Biochemistry and toxicology of the diterpenoid glycoside atractyloside. Food Chem Toxicol. 36:335-346.

Trott O, Olson AJ. 2010. Software news and update AutoDock Vina: improving the speed and accuracy of docking with a new scoring function, efficient optimization, and multithreading. J Comput Chem. 31:455-461.

WHO. 2014. World malaria report 2014. Geneve: WHO. 